\title{
Interpreting Gene Expression Data by Searching for Enriched Gene Sets
}

\author{
Igor Trajkovski ${ }^{1}$ and Nada Lavrać ${ }^{1,2}$ \\ 1 Department of Knowledge Technologies, Jožef Stefan Institute, \\ Jamova 39, Ljubljana, Slovenia \\ 2 University of Nova Gorica, Vipavska 13, Nova Gorica, Slovenia \\ \{igor.trajkovski, nada.lavrac\}@ijs.si
}

\begin{abstract}
This paper presents a novel method integrating gene-gene interaction information and Gene Ontology for the construction of new gene sets that are potentially enriched. Enrichment of a gene set is determined by Gene Set Enrichment Analysis, which is a microarray data analysis method that uses ranks of the genes, according to their differentially expression values, to identify significant biological changes in microarray data sets. The experimental results show that the introduced method improves over existing methods, i.e. that it is capable to find new descriptions of the biology governing the experiments, not detectable by the traditional methods of evaluating the enrichment of predefined gene sets, defined by a single GO term.
\end{abstract}

\section{Introduction}

High-throughput technologies such as DNA microarrays and proteomics are revolutionizing biology and medicine. Global gene expression profiling using microarrays monitors changes in the expression of thousands of genes simultaneously. The large amounts of data acquired must then be reduced or "translated" to a smaller set of genes representing meaningful biological differences between control and test systems and validated in an experimental or clinical setting.

In a typical experiment, mRNA expression profiles are generated for thousands of genes from a collection of samples belonging to one of two classes - for example, tumor vs. normal tissue. The genes can be ordered in a ranked list $L$, according to the difference of expression between the classes. The challenge is to extract the meaning from this list.

A common approach involves focusing on a handful of genes at the top of $L$ (genes showing the largest difference in its expression between the classes), to extract the underlying biology responsible for the phenotypic differences. This approach has a few major limitations:

- After correcting for multiple hypotheses testing, no individual gene may meet the threshold for statistical significance, because the relevant biological differences are small relative to the noise inherent to the microarray technology. 
- The opposite situation, one may be left with a long list of statistically significant genes without any common biological function.

- Single-gene analysis may miss important effects on pathways. Cellular processes often affect sets of genes acting jointly. An increase of $20 \%$ in all genes encoding members of a biological process may dramatically alter the execution of that process, and its impact on other processes, than a 10-fold increase in a single gene.

- It is not a rare case when different groups studying the same biological system, report a list of statistically significant genes from the two studies that have a significantly small overlap.

To overcome these analytical challenges, a recently developed method, called Gene Set Enrichment Analysis (GSEA) [1], can evaluate microarray data at the level of gene sets. Biologically defined sets, for example genes that belong to a pathway or genes known to have the same biological function, are good examples of such gene sets. The most popular choice for gene sets are genes annotated with some term from the Gene Ontology (GO) [2]. The goal of GSEA is to determine whether members of a gene set $S$ tend to occur toward the top of the list $L$, in which case the gene set is correlated with the phenotypic class distinction.

In this work we propose a method for generating new gene sets that have relevant biological interpretations, by combining the existing gene sets, and by inclusion of gene-gene interaction information available from the public gene annotation databases. The experimental results show that our method can find descriptions of interesting enriched gene sets, that traditional methods are unable to discover. We applied the proposed method to three gene expression data sets and we find that significant number of discovered gene sets have description which highlights the underlying biology that is responsible for distinguishing one class from the other classes.

The paper is organized as follows. In Section 2 we give some background information about methods for finding the differentially expressed genes and Gene Set Enrichment Analysis. Section 3 presents the idea of our approach, and the steps taken in the construction of interesting gene sets. Section 4 presents the results of the experiments. In Section 5 we draw some final conclusions.

\section{Background}

\subsection{Differentially expressed genes}

Differentially expressed genes are genes that are expressed differently (relative to the reference) between the conditions of interest. In the context of finding differentially expressed genes, the null hypothesis for each gene is that it is not differentially expressed between two conditions, usually against the twosided alternative hypothesis that the gene is up- or down regulated. The most commonly used statistical test in this setting has been the two-sample t-test [3] [5], although other statistics such as the signal-to-noise ratio [4], or Pearson's correlation [6], have often been used. 
Let $T(g, c)$ denote the t-test score of gene $g$ for a target class $c$, which is computed by the following procedure: $\left[\mu_{1}(g), \sigma_{1}(g)\right]$ and $\left[\mu_{2}(g), \sigma_{2}(g)\right]$ denote the means and standard deviations of the logarithm of the expression levels of gene $g$ for the samples in class $c$ and samples in $C \backslash c$, respectively. Also, let $N_{1}$ $=|c|$ and $N_{2}=|C \backslash c| . T(g, c)$ is computed by the following formula:

$$
T(g, c)=\frac{\mu_{1}(g)-\mu_{2}(g)}{\sqrt{\frac{\sigma_{1}(g)}{N_{1}}+\frac{\sigma_{2}(g)}{N_{2}}}}
$$

which reflects the difference between the classes relative to the standard deviation within the classes. Large values of $|T(g, c)|$ indicate a strong correlation between the gene $g$ and class $c$, while the sign of $T(g, c)$ being positive or negative corresponds to $g$ being more highly expressed in class $c$ or in other classes.

\subsection{Gene Set Enrichment Analysis (GSEA)}

GSEA considers experiments with gene expression profiles from samples belonging to two classes. First, genes are ranked based on the correlation between their expression and the class distinction by using any suitable metric. In our experiments we ranked the genes according to their t-score value.

Given a predefined set of genes $S$ (e.g. genes involved in some biological process) the goal of GSEA is to determine whether the members of $S$ are randomly distributed throughout ranked gene list $L$ or primarily found at the top.

There are two major steps in the GSEA method:

1. Calculation of an Enrichment Score. Enrichment score (ES) reflects the degree to which a set $S$ is overrepresented at the top of the ranked list $L$. The score is calculated by walking down the list $L$, increasing a running-sum statistic when encountering a gene in $S$ and decreasing it when gene is not in $S$. The magnitude of the increment depends on the size of $S$ and the total number of genes $N$. The enrichment score is the maximum deviation from zero encountered in the random walk (see Fig. 1). If $L=\left(g_{1}, g_{2}, \ldots, g_{N}\right)$ is a ranked list of genes, according to their t-score, enrichment score ES is calculated as:

$$
\begin{gathered}
\operatorname{Hit}(S, i)=\sum_{\substack{g_{j} \in S \\
1 \leq j \leq i}} \frac{1}{|S|} \quad \operatorname{Miss}(S, i)=\sum_{\substack{g_{j} \in S \\
1 \leq j \leq i}} \frac{1}{N-|S|} \\
E S(S)=\max _{1 \leq i \leq N}|\operatorname{Hit}(S, i)-\operatorname{Miss}(S, i)|
\end{gathered}
$$

2. Estimation of Significance Level of ES. The statistical significance of the ES is computed by using an empirical phenotype-based permutation test procedure that preserves the complex correlation structure of the gene expression data. Specifically, one permutes the phenotype labels and recomputes the ES of the gene set for the permuted data, which generates a null distribution for the ES. The empirical, p-value of the observed ES is then calculated relative to this null distribution. 

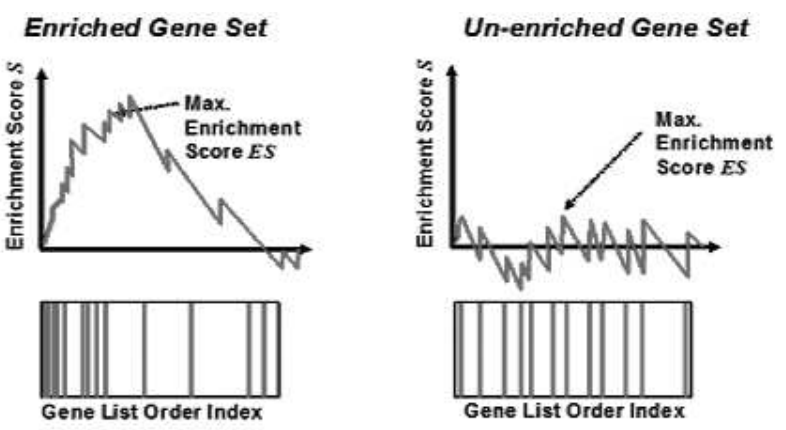

Fig. 1. 'Spectral lines' show the position of gene set members on the ranked gene list. This figure was borrowed from the supplementary material of [1].

\section{Generation of new gene sets}

Methods that test for enrichment of GO terms have been proposed by [7], [8], [9] and [10]. A comparative study of commonly used tools for analyzing GO term enrichment was presented by [11]. [12] presented two novel algorithms that improve GO term scoring using the underlying GO graph topology.

None of the papers includes the gene interaction information, and none of them presents a method for the construction of novel gene sets, but rather they just calculate the enrichment of an a-priory given list of gene sets.

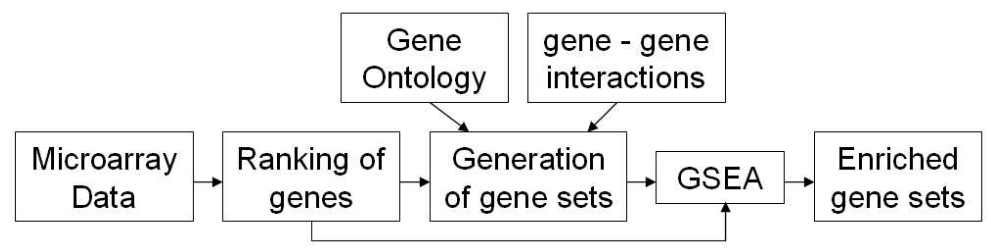

Fig. 2. Data flow of the proposed method for generation of enriched gene sets.

First, let us mention some properties of the gene annotations with GO terms:

- one gene can be annotated with several GO terms,

- a GO term may have thousands of genes annotated to it,

- if a gene is annotated with a GO term A then it is annotated with all ancestors of $\mathrm{A}$.

From this information, we can conclude that each GO term defines one gene set, that one gene can be member of several gene sets, and that some gene sets are subsets of other gene sets.

Second, let Func, Proc and Comp denote the sets of gene sets that are defined by the GO terms that are a subterm of the term "molecular function", "biological process" and "cellular component", respectively. 
Our method relies on two ideas, that are used in the construction of new gene sets:

- Inclusion of gene interaction information. There are cases when some abrupted processes are not detectable by the enrichment score, one reason can be that the genes had a slight increase/decrease in their expression, but had a much larger effect on the interacting genes. Therefore we think that it is reasonable to construct a gene set whose members interact with another gene set.

Formally: if $G_{1} \in$ Func (or Proc, Comp, respectively), then $G_{2}=\left\{g_{2} \mid g_{2}\right.$ is a gene, and $g_{2}$ interacts with $\left.g_{1} \in G_{1}\right\}$ was added to Func (or Proc,Comp).

- Intersection of gene sets. There are cases where two or three given gene sets are not significantly enriched, but their intersection is significantly enriched.

Formally: if $G_{1} \in$ Func, $G_{2} \in$ Proc and $G_{3} \in$ Comp, then $G_{4}=G_{1} \bigcap G_{2} \bigcap G_{3}$ is a new defined gene set.

For example, it can happen that a gene set defined by the molecular function $F$ is not enriched, because a lot of genes in different parts of the cell execute it and one can not expect that all of them will be over/under expressed, but if genes with that function in some specific part of the cell $C_{\text {part }}$ are abnormally active, then it can be elegantly captured by the following gene set:

$$
\text { function }(\mathrm{F}) \bigcap \operatorname{component}\left(C_{\text {part }}\right) \text {. }
$$

Note that all genes are annotated with the top three GO terms: "molecular function", "biological process" and "cellular component", which means that top three GO terms contain all the genes.

The newly defined gene sets are interpreted very intuitively. For example, the gene set defined as intersection of a "functional" term A and "process" term B:

$$
\operatorname{Func}(\mathrm{A}), \operatorname{Proc}(\mathrm{B}) \equiv \operatorname{function}(\mathrm{A}) \bigcap \operatorname{process}(\mathrm{B})
$$

is interpreted as: Genes that are part of the process $B$ and have function $A$, or:

$$
\operatorname{int}(\operatorname{Proc}(\mathrm{A})) \equiv\left\{g_{2} \mid g_{2} \text { is a gene, and } g_{2} \text { interacts with } g_{1} \in \operatorname{Proc}(\mathrm{A})\right\}
$$

is interpreted as: Genes that interact with genes that are members of process A, or:

$$
\operatorname{int}(\operatorname{Proc}(\mathrm{A}), \operatorname{Comp}(\mathrm{B})) \equiv \operatorname{int}(\operatorname{Proc}(\mathrm{A})) \bigcap \operatorname{int}(\operatorname{Comp}(\mathrm{B}))
$$

is interpreted as: Genes that interact with genes that are members of process $A$, and genes that operate in cellular component $B$. 
The number of the newly defined gene sets is huge. In December 2006, |Func $\mid$ $=7513, \mid$ Proc $\mid=12549$ and $|C o m p|=1846$. After the inclusion of the gene interaction information, the size of these sets is doubled. Then the number of newly generated gene sets is:

$$
2^{3} \times \mid \text { Func }|\times| \text { Proc }|\times| \text { Comp } \mid \approx 1.4 \times 10^{12}
$$

For each of these sets we need to compute its enrichment score, ES, that takes linear time in the number of genes $\left(\approx 2 \times 10^{4}\right)$, we get $\approx 3 \times 10^{16}$ floating operations. If we want to statistically validate founded enriched gene sets, usually with 1000 permutation tests, we get $\approx 10^{20}$ operations, that is well above the average performance of today PC's. Therefore we need to efficiently search the space of newly generated gene sets for possible enriched gene sets.

The first idea for improvement is that we are not interested in generating all possible gene sets, but only those that are potentially enriched, and have some minimum number of genes at the top of the list, for example 5 in the first 100 , or 10 in the first 300 genes of the list. That is a weak constraint concerning the biological interpretation of the results, because we are not really interested in the gene sets that do not have this number of genes at the top of the list, but it is a hard constraint concerning the pruning of the search space of all gene sets. By having this constraint we can use the GO topology to efficiently generate all gene sets that satisfy it.

GO is a directed acyclic graph, the root of the graph is the most general term, which means that if one term (gene set) does not satisfy our constraint, than all its descendants will also not satisfy it, because they cover a subset of the genes covered by the given term. In this way we can significantly prune the search space of possible enriched gene sets. Therefore, we first try to construct gene sets from the top nodes of the GO, and if we fail we do not refine the last added term that did not satisfy our constraint.

In the original version of Kolmogorov-Smirnov test, used by GSEA, the ES statistic used equal weights at every step, which yielded high scores for sets clustered near the middle of the ranked list. These sets do not represent biologically relevant correlation with the phenotype. We addressed this issue by weighting the steps according to each genes correlation with a phenotype. Like in the original version we first rank the $N$ genes to form $\mathrm{L}=\left(g_{1}, g_{2}, \ldots, g_{N}\right)$ according to their t-score, $t\left(g_{j}\right)=t_{j}$, of their expression profiles with class $c$. Then the running sum $H i t$ is computed by following formula:

$$
H i t(S, i)=\sum_{\substack{g_{j} \in S \\ 1 \leq j \leq i}} \frac{\left|t_{j}\right|}{\sum_{g_{j} \in S}\left|t_{j}\right|}
$$

Miss, and ES statistic were calculated with the original formulas (2). 


\section{Experiments}

We applied the proposed methodology to three classification problems: leukemia [4], diffuse large B-cell lymphoma (DLBCL) [13] and prostate tumor [14]. All of them are binary classification problems. The leukemia data includes 48 acute lymphoblastic leukemia (ALL) samples and 25 acute myeloid leukemia (AML) samples, each with 7074 gene expression values. The DLBCL data set includes 7070 gene expression profiles for 77 patients, 58 with DLBCL and 19 with follicular lymphoma (FL). The prostate tumor data set includes 12533 genes measured for 52 prostate tumor and 50 normal tissue samples. The data for these three data sets were produced from Affymetrix gene chips and are available at http://www.genome.wi.mit.edu/cancer/. Gene annotations and interaction data was downloaded from Entrez database ftp://ftp.ncbi.nlm.nih.gov/gene/.

Note that this paper does not address the problem of discriminating between the classes. Instead, for the given target class we aim at finding relevant enriched gene sets that can capture the underlying biology characteristic for that class.

\subsection{Experimental results}

To illustrate the straightforward interpretability of the enriched gene sets found by our approach, we provide the best-scoring gene sets for some of the target classes in the mentioned three classification problems (see Tables 1, 2 and 3). We should mention that enriched gene sets that include too general GO terms (i.e. "biological function", "protein binding", "cellular physiological process", "cytoplasm", etc.), were removed from the result list.

Table 1. Summary of GSEA results for the leukemia dataset, with $p$-value $\leq 0.001$.

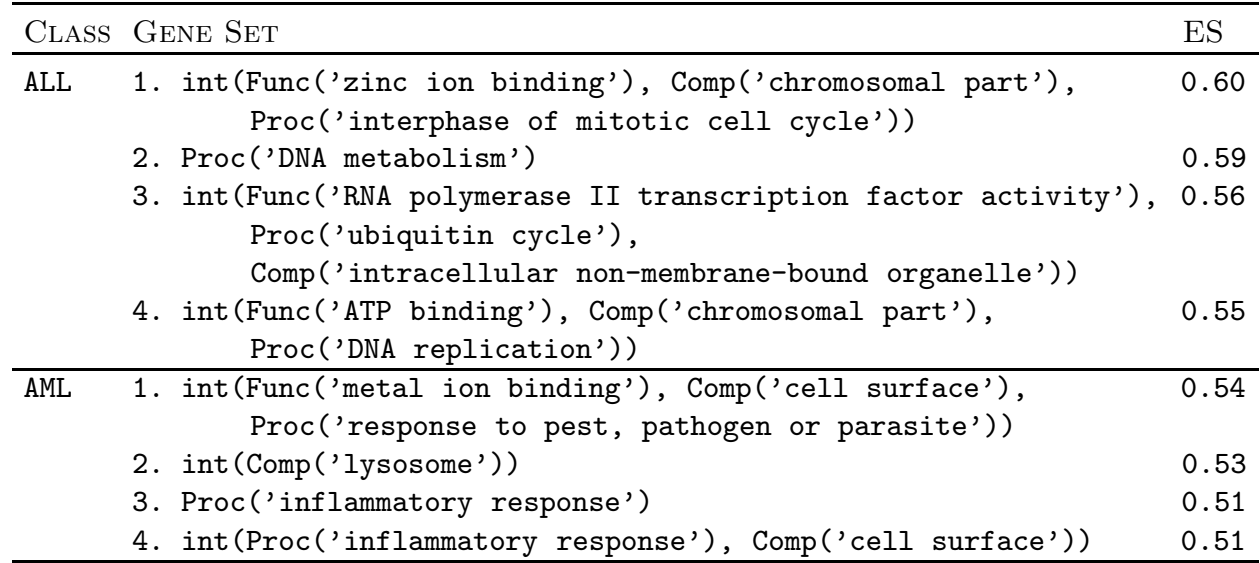


Table 2. Summary of GSEA results for the DLBCL dataset, with $p$-value $\leq 0.001$.

\begin{tabular}{llc}
\hline Class & GEnE SET & ES \\
\hline DLBCL & 1. int(Func('exonuclease activity'), Comp('nucleus')) & 0.62 \\
& 2. int(Func('DNA binding'), Comp('nucleus'), & 0.61 \\
& $\quad$ Proc('regulation of DNA replication') & 0.59 \\
& 3. Proc('DNA replication') & 0.56 \\
& 4. int(Comp('('chromosomal part'), & 0.31 \\
\hline FL & 1. Comp('integral to plasma membrane'), & \\
& Proc('cell surface receptor linked signal transduction') & 0.28 \\
& 2. Comp('integral to membrane'), & \\
\hline
\end{tabular}

Table 3. Summary of GSEA results for the prostate dataset, with $p$-value $\leq 0.001$.

\begin{tabular}{llc}
\hline Class & GENE SET & ES \\
\hline Tumor & 1. Func('structural constituent of ribosome'), & 0.74 \\
& Proc('protein biosynthesis') & \\
& 2. Proc('protein biosynthesis'), Comp('cytoplasmic part') & 0.70 \\
3. int(Func('transitional metal ion binding'), & 0.62 \\
$\quad$ Proc('protein folding'), & \\
$\quad$ Comp('intracellular membrane-bound organelle')) & 0.60 \\
& $\quad$ Proc('regulation of apoptosis')) & \\
\hline 4. int(Func('zinc ion binding'), Comp('cytoplasmic part'), & 0.38 \\
& Comp('membrane')) & 0.35 \\
\hline
\end{tabular}

For comparison of the enrichment of the found gene sets with the gene sets defined by a single GO term, in Table 4 we list the most enriched gene sets defined by a single GO term, for the leukemia dataset. We can see that ES of the single GO terms is much smaller then the ES of the newly constructed gene sets, and most importantly, the found gene sets are constructed from not enriched GO terms. Similar results we got for the other two datasets.

\subsection{Statistical validation}

The following procedure calculated the significance of an observed ES by comparing it with the set of scores $E S_{N U L L}$ computed with randomly assigned phenotypes: 
Table 4. Summary of GSEA results for the leukemia dataset, with $p$-value $\leq 0.005$. Gene sets constructed from a single GO term.

\begin{tabular}{lll}
\hline Class & GENE SET & ES \\
\hline ALL & 1. Proc('DNA metabolism') & 0.59 \\
& 2. Comp ('intracellular non-membrane-bound organelle') & 0.35 \\
& 3. Proc('development') & 0.22 \\
& 4. Comp ('cytoplasmic part') & 0.22 \\
& 4. Proc('transport') & 0.22 \\
\hline AML & 1. Proc('inflammatory response') & 0.51 \\
& 2. Proc('response to chemical stimulus') & 0.41 \\
& 3. Proc('proteolysis') & 0.38 \\
4. Proc('cell communication') & 0.33 \\
\hline
\end{tabular}

1. Randomly assign the original phenotype labels to samples, reorder genes according to their t-score values, and re-compute $\mathrm{ES}(\mathrm{S})$.

2. Repeat step 1 for 1,000 permutations, and create a histogram of the corresponding maximum enrichment scores $E S_{N U L L}$.

3. Estimate the p-value for the $E S$ value of the gene set $\mathrm{S}$ from $E S_{N U L L}$ by using the histogram computed at step 2 . If there was not a case where random labeling of the examples give bigger ES value, then p-value $<0.001$.

We use class labeled permutation because it preserves gene-gene correlations and, thus, provides a more biologically reasonable assessment of significance than would be obtained by permuting genes.

\section{Conclusion}

We addressed the problem of finding enriched functional groups of genes based on gene expression data. We proposed a novel method for integrating the gene interaction information into the construction of new interesting relevant gene sets. The experimental results show that the introduced method improves over existing methods, and we base our conclusion on the following facts:

- ES of the newly constructed sets are higher then the ES of any single GO terms.

- Newly constructed sets are composed of non-enriched GO terms, which means that we are extracting additional biological knowledge that can not be found by single GO term GSEA.

- This method is a generalization of the traditional methods. If we turn-off gene-gene interactions and combination of GO terms, we will get classical single GO term GSEA.

We believe that the strength of the proposed method will be even bigger through the expected increase in both the quality and quantity of gene annotations and gene-gene interaction information in the near future. 


\section{Acknowledgment}

This research was supported by the Slovenian Ministry of Higher Education, Science and Technology. We are grateful to Filip Železný for previous joint work on the use of GO and gene interaction information in the analysis of microarray data which has largely stimulated this work.

\section{References}

1. Subramanian A., et al. (2005) Gene set enrichment analysis: A knowledgebased approach for interpreting genome-wide expression profiles. Proc. Natl. Acad. Sci. of the U.S.A., 102(43):15545-15550.

2. Ashburner M, et al. (2000) Gene ontology: tool for the unification of biology. The Gene Ontology Consortium. Nature Genetics 2000 May;25(1):25-9.

3. Snedecor G. W. and Cochran W. G. (1989) Statistical Methods, Eighth Edition, Iowa State University Press.

4. Golub T. R., Slonim D. K., Tamayo P., Huard C., Gaasenbeek M. et al. (1999). Molecular classification of cancer: Class discovery and class prediction by gene expression monitoring. Science, 286:5439, 531-537.

5. Tsai C. A., Chen Y. J. and Chen J. J. (2003) Testing for differentially expressed genes with microarray data. Nucleic Acids Res 31, e52.

6. Troyanskaya O.G., et al. (2002) Nonparametric methods for identifying differentially expressed genes in microarray data. Bioinformatics 18(11):1454-61.

7. Draghici S., , et al. (2003) Global functional profiling of gene expression. Genomics, 81:98-104.

8. Zeeberg B. R., et al. (2003) GoMiner: a resource for biological interpretation of genomic and proteomic data. Genome Biology, 4(4):R28.

9. Al-Shahrour F., et al. (2004) FatiGO: a web tool for finding significant associations of Gene Ontology terms with groups of genes. Bioinformatics, 20:578-580.

10. Beissbarth T. and Speed T. (2004) GOstat: Find statistically overrepresented Gene Ontologies within a group of genes. Bioinformatics, 1(1):1-2.

11. Khatri P. and Draghici S. (2005) Ontological analysis of gene expression data: current tools, limitations, and open problems. Bioinformatics, 21(18):3587-3595.

12. Alexa A., et al. (2006) Improved Scoring of Functional Groups from Gene Expression Data by Decorrelating GO Graph Structure. Bioinformatics, 22(13):1600-1607.

13. Shipp M.A., Ross K.N., Tamayo P., et al. (2002) Diffuse large b-cell lymphoma outcome prediction by gene-expression profiling and supervised machine learning. Nature Medicine 8:6874

14. Singh D., Febbo P.G., Ross K., et al. (2002) Gene expression correlates of clinical prostate cancer behavior. Cancer Cell 1 (2002):203209 